\title{
THE LATTICE OF PSEUDOVARIETIES OF INVERSE SEMIGROUPS
}

\author{
T. E. Hall and K. G. Johnston
}

\begin{abstract}
As introduced by Eilenberg and Schützenberger, a pseudovariety is a class of finite algebras closed under the formation of homomorphic images, subalgebras and direct products of finitely many algebras. Many previous results about the lattice of varieties of inverse semigroups are found to have analogues for the lattice of pseudovarieties of (finite) inverse semigroups. In particular, certain intervals are modular, including the interval consisting of the pseudovarieties of groups.
\end{abstract}

1. Introduction and summary. A pseudovariety, introduced by Eilenberg and Schützenberger [8], is a class of finite algebras closed under forming homomorphic images, subalgebras, and finite direct products (each class of algebras considered will be assumed to consist of algebras all of the same type). As usual, we consider inverse semigroups to have two operations, that of multiplication, and that of inversion, so that just as the class $\mathscr{I}$ of all inverse semigroups is a variety, the class of all finite inverse semigroups $\mathscr{I}_{F}$ is a pseudovariety.

Finite semigroups have long been a part of language and automata theory (Eilenberg [7], Lallement [13]), since each recognizable language has a finite syntactic semigroup and each automaton has a finite action semigroup. Not only do pseudovarieties give a natural way of classifying finite algebras generally, but also pseudovarieties of semigroups are in a natural one-to-one correspondence with varieties of recognizable (or rational) languages [7, Vol. B, Theorem 3.4s].

Finite inverse semigroups and injective (or reversible) automata are now also studied in automata theory, for example in $[10,12,14,15$, $16,19,21]$. The pseudovariety of semigroups generated by $\mathscr{I}_{F}$ was shown by Ash [3] to be the pseudovariety of (finite) semigroups with all idempotents commuting, which we denote by $\mathscr{S}_{i c, F}$. The variety of languages corresponding to $\mathscr{S}_{i c, F}$ has been described by Margolis and Pin ([15, Theorem 5.2] and [16]). Of course each subpseudovariety of $\mathscr{S}_{i c, F}$ also corresponds to a variety of languages, and this correspondence has in part been studied by Ash, Hall and Pin [4]. Consider a map $\alpha: \mathscr{L}_{s p v}\left(\mathscr{S}_{i c, F}\right) \rightarrow \mathscr{L}_{p v}\left(\mathscr{I}_{F}\right)$, given by $\mathscr{P}_{\alpha}=\mathscr{P} \cap \mathscr{I}_{F}$ (here $\mathscr{L}_{\text {spv }}\left(\mathscr{S}_{i c, F}\right)$ denotes the lattice of (semigroup) pseudovarieties 
contained in $\mathscr{S}_{i c, F}$, and $\mathscr{L}_{p v}\left(\mathscr{I}_{F}\right)$ denotes the lattice of (inverse semigroup) pseudovarieties contained in $\mathscr{F}_{F}$ ). This $\alpha$ is easily shown to be a complete lattice homomorphism from the following well known results: (i) $\operatorname{Reg}(S)$, the set of regular elements of a semigroup $S$, is an inverse subsemigroup of $S$ if the idempotents of $S$ commute; and (ii) for any morphism $\varphi: S \rightarrow T$ of a finite semigroup $S$ onto a semigroup $T$, each regular element of $T$ is an image of a regular element of $S$ [22, Proposition 3.2(d)], that is, $\operatorname{Reg}(S) \varphi=\operatorname{Reg}(T)$ (since trivially $\operatorname{Reg}(S) \varphi \subseteq \operatorname{Reg}(T))$. Each $\alpha \circ \alpha^{-1}$ class is thus a complete sublattice, and hence an interval, of $\mathscr{L}_{s p v}\left(\mathscr{S}_{i c, F}\right)$; in fact we can easily see that for each $\mathscr{P} \in \mathscr{L}_{p v}\left(\mathscr{I}_{F}\right)$ we have $\mathscr{P} \alpha \circ \alpha^{-1}=\left[\langle\mathscr{P}\rangle_{s p v},\langle\mathscr{P}\rangle_{\text {max }}\right]$, where $\langle\mathscr{P}\rangle_{s p v}$ (or $\langle\mathscr{P}\rangle_{\min }$ ) say, is the semigroup pseudovariety generated by $\mathscr{P}$, and

$$
\langle\mathscr{P}\rangle_{\max }=\left\{S \in \mathscr{S}_{i c, F}: \operatorname{Reg}(S) \in \mathscr{P}\right\} .
$$

Since $\mathscr{L}_{s p v}\left(\mathscr{S}_{i c, F}\right) /\left(\alpha \circ \alpha^{-1}\right) \cong \mathscr{L}_{p v}\left(\mathscr{J}_{F}\right)$, our study of $\mathscr{L}_{p v}\left(\mathscr{I}_{F}\right)$ is a step in the study of $\mathscr{L}_{s p v}\left(\mathscr{S}_{i c, F}\right)$ and the corresponding lattice of varieties of languages.

After preliminaries in $\S 2$, we consider in $\S 3$ the lattice of pseudovarieties of groups, $\mathscr{L}_{p v}\left(\mathscr{G}_{F}\right)$, obtaining a monomorphism into the lattice of $\mathscr{L}_{g v}(\mathscr{G})$ of generalized varieties of groups, and we deduce $\mathscr{L}_{p v}\left(\mathscr{G}_{F}\right)$ is modular.

In $\S 4$, joins with the pseudovariety $\mathscr{G}_{F}$ of groups are considered, and the map $\phi: \mathscr{L}_{p v}\left(\mathscr{I}_{F}\right) \rightarrow\left[\mathscr{G}_{F}, \mathscr{L}_{p v}\left(\mathscr{I}_{F}\right)\right]$, given by $\mathscr{P} \phi=\mathscr{P} \vee \mathscr{G}_{F}$ for each $\mathscr{P} \in \mathscr{L}_{p v}\left(\mathscr{I}_{F}\right)$, is shown to be a complete lattice morphism, and in $\S 5$ the intervals $\mathscr{P} \phi \circ \phi^{-1}$ are characterized and shown to be modular.

In $\S 6$, meets with $\mathscr{G}_{F}$ are considered, and the map $\psi: \mathscr{L}_{p v}\left(\mathscr{I}_{F}\right) \rightarrow$ $\mathscr{L}_{p v}\left(\mathscr{G}_{F}\right)$, given by $\mathscr{P} \psi=\mathscr{P} \cap \mathscr{G}_{F}$ for each $\mathscr{P} \in \mathscr{L}_{p v}\left(\mathscr{I}_{F}\right)$, is shown to be a complete lattice morphism.

In $\S 7$, the lowest three intervals of $\S 5$ are shown to form a sublattice isomorphic to the direct product of $\mathscr{L}_{p v}\left(\mathscr{G}_{F}\right)$ and a three-element chain, and so in particular forms a modular lattice.

We omit those proofs which are similar to proofs of the corresponding results for varieties of inverse semigroups (as in the text [18] by Petrich), indicating what modifications suffice to yield our results.

\section{Preliminaries.}

Result 2.1 ([9, Theorem 5] and [11, Theorem V.3.2]). The maximum congruence contained in Green's relation $\mathscr{H}$ on any regular semi- 
group $S, \mu=\mu(S)$ say, is given by

$\mu=\{(a, b) \in \mathscr{H}:$ for some [for each pair of] $\mathscr{H}$-related inverses $a^{\prime}$ of $a$ and $b^{\prime}$ of $b, a^{\prime} e a=b^{\prime} e b$ for each idempotent $\left.e \leq a a^{\prime}\right\}$.

If $S$ is an inverse semigroup, then

$\mu=\left\{(a, b) \in S \times S: a^{-1} e a=b^{-1} e b\right.$ for each idempotent $\left.e \in S\right\}$.

For congruences $\rho, \sigma$ on algebras $S, T$ respectively, define a congruence $\rho \times \sigma$ on the direct product $S \times T$ by, for all $\left(s_{1}, t_{1}\right),\left(s_{2}, t_{2}\right) \in S \times T$, $\left(s_{1}, t_{1}\right)(\rho \times \sigma)\left(s_{2}, t_{2}\right)$ if and only if $s_{1} \rho s_{2}$ and $t_{1} \sigma t_{2}$.

The following result can now be routinely proved from Result 2.1.

Result 2.2. For any regular semigroups $S$ and $T$,

(i) $\mu(S \times T)=\mu(S) \times \mu(T)$, and

(ii) the map $(S \times T) / \mu \rightarrow(S / \mu) \times(T / \mu)$ given by $(s, t) \mu \mapsto(s \mu, t \mu)$ is (well-defined and) an isomorphism.

Following Ash [2], by a generalized variety we mean a class of algebras closed under the operations of forming all homomorphic images, all subalgebras, all products of finitely many algebras, and all powers (and we denote these operations by $H, S, P_{f}$, Pow respectively). It is easily checked [2, Theorem 1 and Comments] that the generalized variety $\langle\mathscr{K}\rangle_{g v}$, generated by any class $\mathscr{K}$ of algebras, is given by $\langle\mathscr{K}\rangle_{g v}=H S P_{f} \operatorname{Pow}(\mathscr{K})$.

We denote by $\mathscr{F}$ in the class of all finite algebras. For any class $\mathscr{K}$ of algebras, $\langle\mathscr{K}\rangle_{v}$, and $\langle\mathscr{K}\rangle_{p v}$ denote the variety and the pseudovariety (when $\mathscr{K} \subseteq \mathscr{F}$ in) respectively, generated by $\mathscr{K}$.

Result 2.3 (implicit in [2]). For any finite set $\mathscr{K}$ of finite algebras,

(i) $\langle\mathscr{K}\rangle_{g v}=\langle\mathscr{K}\rangle_{v}$,

(ii) $\langle\mathscr{K}\rangle_{p v}=\langle\mathscr{K}\rangle_{v} \cap \mathscr{F i n}$.

Proof. (i) $\langle\mathscr{K}\rangle_{g v}=\operatorname{HSP}_{f} \operatorname{Pow}(\mathscr{K})=\operatorname{HSP}(\mathscr{K})=\langle\mathscr{K}\rangle_{v}$.

(ii) From [2, Theorem 2], $\langle\mathscr{K}\rangle_{p v}=\langle\mathscr{K}\rangle_{g v} \cap \mathscr{F}$ in, so from (i), $\langle\mathscr{K}\rangle_{p v}=$ $\langle\mathscr{K}\rangle_{v} \cap \mathscr{F}$ in.

Each pseudovariety is determined by an $\omega$-sequence of identities [8], in the sense that for each pseudovariety $\mathscr{P}$ there is an $\omega$-sequence $\left[u_{i}=v_{i}\right]_{i \in \mathbf{N}}$ (where $\left.\mathbf{N}=\{1,2,3, \ldots\}\right)$ of identities such that a finite algebra $A$ is in $\mathscr{P}$ if and only if $A$ ultimately satisfies $\left[u_{i}=v_{i}\right]_{i \in \mathbf{N}}$ (in the sense that there exists $n \in \mathbf{N}$ such that $A$ satisfies $u_{i}=v_{i}$ for all $i \geq n$ ), and conversely, for each $\omega$-sequence of identities, the 
class of those finite algebras that ultimately satisfy the sequence is a pseudovariety.

We denote by $\left[u_{i}=v_{i}\right]_{i \in \mathbf{N}}^{p v}$ the pseudovariety so determined by $\left[u_{i}=v_{i}\right]_{i \in \mathbf{N}}$.

An important pseudovariety of inverse semigroups, with no variety counterpart, is $\mathscr{C}_{F}$, the class of finite combinatorial (or $\mathscr{C}$-trivial) inverse semigroups. Clearly, $\mathscr{C}_{F}$ is given, within $\mathscr{I}_{F}$, by $\mathscr{C}_{F}=$ $\left[x^{i+1}=x^{i}\right]_{i \in \mathbf{N}}^{p v}$.

3. Pseudovarieties of groups. We show that there is a monomorphism from the lattice $\mathscr{L}_{p v}\left(\mathscr{G}_{F}\right)$ of pseudovarieties of groups into the lattice $\mathscr{L}_{g v}(\mathscr{G})$ of generalized varieties of groups, which (from Ash's results [2, Theorem 1 and Comments]) is isomorphic to the lattice $\mathscr{S L}_{v}(\mathscr{G})$ of ideals of the lattice $\mathscr{L}_{v}(\mathscr{G})$ of varieties of groups. By Crawley and Dilworth $[6,9.1]$, the lattice of ideals of a lattice $L$ satisfies the same identities as the lattice $L$, so we will obtain that $\mathscr{L}_{p v}\left(\mathscr{G}_{F}\right)$ satisfies each identity satisfied by $\mathscr{L}_{v}(\mathscr{G})$, and so, in particular, is modular.

For any variety $\mathscr{V}$, it is natural to consider the map Gen from the lattice $\mathscr{L}_{p v}(\mathscr{V})$ of pseudovarieties contained in $\mathscr{V}$ to the lattice $\mathscr{L}_{g v}(\mathscr{V})$ of generalized varieties contained in $\mathscr{V}$, which maps each $\mathscr{P} \in \mathscr{L}_{p v}(\mathscr{V})$ to $\operatorname{Gen}(\mathscr{P})=\langle\mathscr{P}\rangle_{g v}$, the generalized variety generated by $\mathscr{P}$. From [2, Theorem 2] it follows that $\operatorname{Gen}(\mathscr{P}) \cap \mathscr{F}_{\text {in }}=\mathscr{P}$, so the map Gen is one-to-one. For any $\mathscr{P}, \mathscr{Q} \in \mathscr{L}_{p v}(\mathscr{V})$, it is easily seen that $\operatorname{Gen}\left(\mathscr{P} \vee_{p v} \mathscr{Q}\right)=\operatorname{Gen}(\mathscr{P}) \vee_{g v} \operatorname{Gen}(\mathscr{Q})$ (where $\vee_{p v}$ denotes join in $\mathscr{L}_{p v}(\mathscr{V})$ and $\vee_{g v}$ denotes join in $\left.\mathscr{L}_{g v}(\mathscr{V})\right)$; that is, Gen: $\mathscr{L}_{p v}(\mathscr{V}) \rightarrow$ $\mathscr{L}_{g v}(\mathscr{V})$ is a $\vee$-morphism. C. J. Ash found a necessary and sufficient condition for Gen to be an $\cap$-morphism.

Lemma 3.1 (C. J. Ash, private communication). For any variety $\mathscr{V}$, the following are equivalent:

(i) for all $A, B \in \mathscr{V} \cap \mathscr{F}$ in there exists $C \in \mathscr{V} \cap \mathscr{F}$ in such that $\langle A\rangle_{v} \cap\langle B\rangle_{v}=\langle C\rangle_{v}$

(ii) for all $\mathscr{P}, \mathscr{Q} \in \mathscr{L}_{p v}(\mathscr{V})$, $\operatorname{Gen}(\mathscr{P}) \cap \operatorname{Gen}(\mathscr{Q})=\operatorname{Gen}(\mathscr{P} \cap \mathscr{Q})$.

Proof. (i) $\Rightarrow($ ii). Take any algebra $X \in \operatorname{Gen}(\mathscr{P}) \cap \operatorname{Gen}(\mathscr{Q})$. Then there is a finite subset $\mathscr{A}$ of $\mathscr{P}$ such that $X \in\langle\mathscr{A}\rangle_{v}$, whence $X \in\langle A\rangle_{v}$, where $A$ is the direct product of the algebras in $\mathscr{A}$. Likewise there exists a finite algebra $B \in \mathscr{Q}$ such that $X \in\langle B\rangle_{v}$. From condition (i), $\langle A\rangle_{v} \cap\langle B\rangle_{v}=\langle C\rangle_{v}$ for some

$$
C \in\langle A\rangle_{v} \cap\langle B\rangle_{v} \cap \mathscr{F i n}_{i n}=\langle A\rangle_{p v} \cap\langle B\rangle_{p v} \subseteq \mathscr{P} \cap \mathscr{Q}
$$

from Result 2.3. 
Thus $X \in\langle C\rangle_{v} \subseteq \operatorname{Gen}(\mathscr{P} \cap \mathscr{Q})$, so $\operatorname{Gen}(\mathscr{P}) \cap \operatorname{Gen}(\mathscr{Q}) \subseteq \operatorname{Gen}(\mathscr{P} \cap \mathscr{Q})$. The reverse containment is trivial.

(ii) $\Rightarrow\left(\right.$ i). Take any finite algebras $A, B \in \mathscr{V}$ and put $\mathscr{P}=\langle A\rangle_{p v}$ and $\mathscr{Q}=\langle B\rangle_{p v}$.

By condition (ii), $\operatorname{Gen}(\mathscr{P}) \cap \operatorname{Gen}(\mathscr{Q})=\operatorname{Gen}(\mathscr{P} \cap \mathscr{Q})$. As in [2], $\operatorname{Gen}(\mathscr{P})=\operatorname{Gen}(A)=\operatorname{HSP}_{\mathrm{f}} \operatorname{Pow}(A)=\operatorname{HSP}(A)=\langle A\rangle_{v}$; and likewise $\operatorname{Gen}(\mathscr{Q})=\langle B\rangle_{v}$, so $\langle A\rangle_{v} \cap\langle B\rangle_{v}=\operatorname{Gen}(\mathscr{P} \cap \mathscr{Q})$.

Let $F_{\omega}$ be the free algebra on countably many generators in the variety $\langle A\rangle_{v} \cap\langle B\rangle_{v}=\operatorname{Gen}(\mathscr{P} \cap \mathscr{Q})=\operatorname{HSP}_{f} \operatorname{Pow}(\mathscr{P} \cap \mathscr{Q})$. Then there exists a finite subset $\mathscr{K}$ of $\mathscr{P} \cap \mathscr{Q}$ such that

$$
F_{\omega} \in \operatorname{HSP}_{f} \operatorname{Pow}(\mathscr{K})=\operatorname{HSP}(\mathscr{K})=\langle\mathscr{K}\rangle_{v}=\langle C\rangle_{v},
$$

where $C$ is the direct product of all the algebras in $\mathscr{K}$. Now

$$
C \in \mathscr{P} \cap \mathscr{Q}=\langle A\rangle_{p v} \cap\langle B\rangle_{p v} \subseteq\langle A\rangle_{v} \cap\langle B\rangle_{v}=\left\langle F_{\omega}\right\rangle_{v} \subseteq\langle C\rangle_{v},
$$

so $\langle A\rangle_{v} \cap\langle B\rangle_{v}=\langle C\rangle_{v}$ as required.

Lemma 3.2. For any finite groups $A, B$, there exists a finite group $C$ such that $\langle A\rangle_{v} \cap\langle B\rangle_{v}=\langle C\rangle_{v}$.

Proof. First recall from Neumann [17, Definition 51.31] that a finite group $A$ is called critical if it does not belong to the variety generated by its proper factors. Recall [17, Definition 51.51$]$ that a group variety $\mathscr{V}$ is called a Cross variety if (i) $\mathscr{V}$ is locally finite, (ii) the laws of $\mathscr{V}$ are finitely based, and (iii) the number of non-isomorphic critical groups in $\mathscr{V}$ is finite.

Then the Oates and Powell theorem [17, Theorem 52.11] states that the variety generated by a finite group is a Cross variety. Thus $\langle A\rangle_{v}$ and $\langle B\rangle_{v}$ are Cross varieties, so by [17, Theorem 51.52] we have that the subvariety $\langle A\rangle_{v} \cap\langle B\rangle_{v}$ is a Cross variety; let $C$ be the direct product of the finitely many non-isomorphic critical groups in $\langle A\rangle_{v} \cap\langle B\rangle_{v}$. By [17, Remark 51.41], $\langle A\rangle_{v} \cap\langle B\rangle_{v}$ is generated by its critical groups, whence $\langle A\rangle_{v} \cap\langle B\rangle_{v}=\langle C\rangle_{v}$, giving the lemma.

THEOREM 3.3. The map Gen: $\mathscr{L}_{p v}\left(\mathscr{G}_{F}\right) \rightarrow \mathscr{L}_{g v}(\mathscr{G})$, given by $\operatorname{Gen}(\mathscr{P})$ $=\langle\mathscr{P}\rangle_{g v}$, is a monomorphism. Thus $\mathscr{L}_{p v}\left(\mathscr{G}_{F}\right)$ satisfies each identity satisfied by $\mathscr{L}_{v}(\mathscr{G})$, and in particular is modular.

Proof. The first statement follows from Lemmas 3.1 and 3.2. The second statement follows from the first, as was shown at the beginning of this section. 
4. Joins with the pseudovariety of groups. We denote by $\mathscr{G}_{F}$ and $\mathscr{F}_{F}$ the pseudovariety of all finite groups and the class of all finite, fundamental inverse semigroups, respectively, while $\mathscr{F}$ denotes the class of all fundamental inverse semigroups.

Theorem 4.1. For any pseudovarieties $\mathscr{P}, \mathscr{Q}$ of inverse semigroups

$$
\mathscr{P} \vee \mathscr{G}_{F} \subseteq \mathscr{Q} \vee \mathscr{G}_{F} \Leftrightarrow \mathscr{P} \cap \mathscr{F}_{F} \subseteq \mathscr{Q} \cap \mathscr{F}_{F}
$$

Proof. Recall that a finite inverse semigroup is covered by a finite $E$-unitary inverse semigroup (the construction in the proof of [18, Lemma VII.4.4] preserves finiteness), and recall that hence any finite inverse semigroup is covered by a subdirect product of $S / \mu$ and a finite group $G$ (the proof of [18, Theorem VII.4.8] also yields this result for finite inverse semigroups).

The proof of Kleinman's result [18, Theorem XII.2.4] for varieties now yields Theorem 4.1.

COROLlaRY 4.2. For any pseudovarieties $\mathscr{P}, \mathscr{Q}$ of inverse semigroups,

$$
\mathscr{P} \vee \mathscr{G}_{F}=\mathscr{Q} \vee \mathscr{G}_{F} \Leftrightarrow \mathscr{P} \cap \mathscr{F}_{F}=\mathscr{Q} \cap \mathscr{F}_{F} .
$$

COROllary 4.3. For any pseudovariety $\mathscr{P}$ of inverse semigroups,

$$
\mathscr{P} \vee \mathscr{G}_{F}=\left\{S \in \mathscr{I}_{F} \mid S / \mu \in \mathscr{P}\right\} .
$$

Proof. Together with the result that a finite inverse semigroup $S$ is covered by a subdirect product of $S / \mu$ and a finite group $G$, the proof of [18, Corollary XII2.6] also proves Corollary 4.3.

For any class $\mathscr{K}$ of inverse semigroups we define

$$
\mathscr{K} / \mu=\{S / \mu \mid S \in \mathscr{K}\} .
$$

COROLlaRY 4.4. (i) For any class $\mathscr{K}$ of finite inverse semigroups, $\langle\mathscr{K}\rangle_{p v} \cap \mathscr{F}_{F}=\langle\mathscr{K} \mid \mu\rangle_{p v} \cap \mathscr{F}_{F}$

(ii) For any class $\mathscr{K}$ of inverse semigroups $\langle\mathscr{K}\rangle_{v} \cap \mathscr{F}=\langle\mathscr{K} \mid \mu\rangle_{v} \cap \mathscr{F}$.

Proof. (i) Trivially, $\langle\mathscr{K} / \mu\rangle_{p v} \cap \mathscr{F}_{F} \subseteq\langle\mathscr{K}\rangle_{p v} \cap \mathscr{F}_{F}$. Take any $S \in \mathscr{K}$. Since $S / \mu \in \mathscr{K} / \mu$ we have $S \in\langle\mathscr{K} / \mu\rangle_{p v} \vee \mathscr{G}_{F}$, by Corollary 4.3. Hence $\langle\mathscr{K}\rangle_{p v} \subseteq\langle\mathscr{K} / \mu\rangle_{p v} \vee \mathscr{G}_{F}$ and so $\langle\mathscr{K}\rangle_{p v} \vee \mathscr{G}_{F} \subseteq\langle\mathscr{K} / \mu\rangle_{p v} \vee \mathscr{G}_{F}$, and then by Theorem 4.1 we have $\langle\mathscr{K}\rangle_{p v} \cap \mathscr{F}_{F} \subseteq\langle\mathscr{K} \mid \mu\rangle_{p v} \cap \mathscr{F}_{F}$, giving equality, as required. 
(ii) An entirely similar proof to the proof of (i), using [18, Theorem XII.2.4 and Corollary XII.2.6], gives statement (ii).

COROLlaRY 4.5. For any pseudovariety $\mathscr{P}$ of inverse semigroups, if $\mathscr{P}$ is determined by the $\omega$-sequence of identities $\left[u_{i}=v_{i}\right]_{i \in \mathbf{N}}$ say, then $\mathscr{P} \vee \mathscr{G}_{F}$ is determined by the $\omega$-sequence $\left[u_{i}^{-1} t_{i}^{-1} t_{i} u_{i}=v_{i}^{-1} t_{i}^{-1} t_{i} v_{i}\right]_{i \in \mathbf{N}}$, where each $t_{i}$ is not in the content of $u_{i}$ and of $v_{i}$.

Proof. Simple modifications of the proof of [18, Corollary XII.2.7] (for example, replacing "satisfies $u_{\alpha}=v_{\alpha}$ for all $\alpha \in A$ " by "satisfies $u_{i}=v_{i}$ for all $i \geq n$, for some $n$ ") gives a proof of Corollary 4.5.

THEOREM 4.6. The mapping $\phi: \mathscr{L}_{p v}\left(\mathscr{I}_{F}\right) \rightarrow\left[\mathscr{G}_{F}, \mathscr{L}_{p v}\left(\mathscr{J}_{F}\right)\right]$ defined by $\mathscr{P} \phi=\mathscr{P} \vee \mathscr{G}_{F}$ for each $\mathscr{P} \in \mathscr{L}_{p v}\left(\mathscr{I}_{F}\right)$, is a complete lattice morphism. The $\phi \circ \phi^{-1}$ congruence class containing any $\mathscr{P} \in \mathscr{L}_{p v}\left(\mathscr{I}_{F}\right)$ is given by

$$
\mathscr{P} \phi \circ \phi^{-1}=\left[\left\langle\mathscr{P} \cap \mathscr{F}_{F}\right\rangle_{p v}, \mathscr{P} \vee \mathscr{G}_{F}\right]
$$

Proof. For any indexed set $\left\{\mathscr{P}_{i} \mid i \in I\right\}$ of pseudovarieties of inverse semigroups we have that trivially

$$
\left(\bigvee_{i \in I} \mathscr{P}_{i}\right) \vee \mathscr{G}_{F}=\bigvee_{i \in I}\left(\mathscr{P}_{i} \vee \mathscr{G}_{F}\right)
$$

and, from Corollary 4.3 we have that

$$
\begin{aligned}
\left(\bigcap_{i \in I} \mathscr{P}_{i}\right) \vee \mathscr{G}_{F} & =\left\{S \in \mathscr{F}_{F} \mid S / \mu \in \bigcap_{i \in I} \mathscr{P}_{i}\right\} \\
& =\bigcap_{i \in I}\left\{S \in \mathscr{J}_{F} \mid S / \mu \in \mathscr{P}_{i}\right\}=\bigcap_{i \in I}\left(\mathscr{P}_{i} \vee \mathscr{G}_{F}\right),
\end{aligned}
$$

so $\phi$ is a complete lattice morphism.

Part of the proof of [18, Theorem XII.2.8] is easily changed to prove that $\mathscr{P} \phi \circ \phi^{-1}=\left[\left\langle\mathscr{P} \cap \mathscr{F}_{F}\right\rangle_{p v}, \mathscr{P} \vee \mathscr{G}_{F}\right]$, but the following is slightly simpler. Take any $\mathscr{Q} \in \mathscr{P} \phi \circ \phi^{-1}$; then $\mathscr{P} \phi=\mathscr{Q} \phi$, that is, $\mathscr{P} \vee \mathscr{G}_{F}=\mathscr{Q} \vee \mathscr{G}_{F}$, whence $\mathscr{P} \cap \mathscr{F}_{F}=\mathscr{Q} \cap \mathscr{F}_{F}$ from Corollary 4.2. Thus

$$
\left\langle\mathscr{P} \cap \mathscr{F}_{F}\right\rangle=\left\langle\mathscr{Q} \cap \mathscr{F}_{F}\right\rangle \subseteq \mathscr{Q} \subseteq \mathscr{Q} \vee \mathscr{G}_{F}=\mathscr{P} \vee \mathscr{G}_{F},
$$

whence $\mathscr{Q} \in\left[\left\langle\mathscr{P} \cap \mathscr{F}_{F}\right\rangle_{p v}, \mathscr{P} \vee \mathscr{F}_{F}\right]$, and so $\mathscr{P} \phi \circ \phi^{-1} \subseteq\left[\left\langle\mathscr{P} \cap \mathscr{F}_{F}\right\rangle_{p v}\right.$, $\left.\mathscr{P} \vee \mathscr{F}_{F}\right]$.

To prove the converse we first note that $\left\langle\mathscr{P} \cap \mathscr{F}_{F}\right\rangle_{p v} \cap \mathscr{F}_{F}=$ $\mathscr{P} \cap \mathscr{F}_{F}$, whence $\left\langle\mathscr{P} \cap \mathscr{F}_{F}\right\rangle_{p v} \vee \mathscr{G}_{F}=\mathscr{P} \vee \mathscr{G}_{F}$ by Corollary 4.2, and 
that trivially $\left(\mathscr{P} \vee \mathscr{G}_{F}\right) \vee \mathscr{G}_{F}=\mathscr{P} \vee \mathscr{G}_{F}$; thus $\left\langle\mathscr{P} \cap \mathscr{F}_{F}\right\rangle_{p v} \in \mathscr{P} \phi \circ \phi^{-1}$ and $\mathscr{P} \vee \mathscr{G}_{F} \in \mathscr{P} \phi \circ \phi^{-1}$, and since a congruence class of a lattice contains the interval between any comparable pair that it contains, we have $\left[\left\langle\mathscr{P} \cap \mathscr{F}_{F}\right\rangle_{p v}, \mathscr{P} \vee \mathscr{G}_{F}\right] \subseteq \mathscr{P} \phi \circ \phi^{-1}$. Thus $\left[\left\langle\mathscr{P} \cap \mathscr{F}_{F}\right\rangle_{p v}, \mathscr{P} \vee \mathscr{G}_{F}\right]=\mathscr{P} \phi \circ \phi^{-1}$, as required.

5. Modularity of certain intervals. We show that for each $\mathscr{P} \in$ $\mathscr{L}_{p v}\left(\mathscr{I}_{F}\right)$, the interval $\left[\left\langle\mathscr{P} \cap \mathscr{F}_{F}\right\rangle_{p v}, \mathscr{P} \vee \mathscr{G}_{F}\right]$ is modular. The corresponding result for varieties, due to Reilly [20] (see also [18, Theorem XII.2.8]), was proved from the fact that the idempotent-separating congruences on a regular semigroup form a modular lattice, by using idempotent-separating, fully invariant congruences on a relatively free inverse semigroup, for which there seems to be no counterpart in pseudovarieties. We prove our result from Reilly's result.

By a locally finite variety we mean a variety in which each member is locally finite (that is, in which each finite subset of each member generates a finite subalgebra). For clarity, we sometimes use the symbols $\vee_{v}$ and $\vee_{p v}$ for the join of varieties and the join of pseudovarieties respectively.

Lemma 5.1. For any locally finite varieties $\mathscr{V}$ and $\mathscr{W}$,

$$
\left(\mathscr{V} \vee_{v} \mathscr{W}\right) \cap \mathscr{F i n}=\left(\mathscr{V} \cap \mathscr{F}_{i n}\right) \vee_{p v}\left(\mathscr{W} \cap \mathscr{F}_{i n}\right)
$$

Proof. The containment of the class on the right in the class on the left is trivial.

So take any $A \in \mathscr{V} \vee_{v} \mathscr{W}$. Then there exist $V \in \mathscr{V}$ and $W \in \mathscr{W}$, a subdirect product $B$ of $V$ and $W$, and a morphism $\phi: B \rightarrow A$ of $B$ onto $A$ (it follows that $A$, and thence $\mathscr{V} \vee_{v} \mathscr{W}$, are locally finite).

Now suppose further that $A \in\left(\mathscr{V} \vee_{v} \mathscr{W}\right) \cap \mathscr{F i n}$. For each $a \in A$ choose $b_{a} \in a \varphi^{-1}$; then without loss of generality $B$ is generated by the finite set $\left\{b_{a}: a \in A\right\}$, whence $B$ is finite. Hence $V$ and $W$ are finite, whence $V \in \mathscr{V} \cap \mathscr{F}_{i n}, W \in \mathscr{W} \cap \mathscr{F}_{i n}$ and so $A=B \varphi \in(\mathscr{V} \cap$ $\left.\mathscr{F}_{i n}\right) \vee_{p v}(\mathscr{W} \cap \mathscr{F} i n)$. Thus $\left(\mathscr{V} \vee_{v} \mathscr{W}\right) \cap \mathscr{F} i n \subseteq\left(\mathscr{V} \cap \mathscr{F}_{i n}\right) \vee_{p v}\left(\mathscr{W} \cap \mathscr{F}_{i n}\right)$ which gives equality as required.

TheOREM 5.2. For each $\mathscr{P} \in \mathscr{L}_{p v}\left(\mathscr{I}_{F}\right)$, the interval $\left[\left\langle\mathscr{P} \cap \mathscr{F}_{F}\right\rangle_{p v}\right.$, $\left.\mathscr{P} \vee \mathscr{G}_{F}\right]$ is modular.

Proof. Take any $\mathscr{X}, \mathscr{Y}, \mathscr{Z} \in\left[\left\langle\mathscr{P} \cap \mathscr{F}_{F}\right\rangle_{p v}, \mathscr{P} \vee \mathscr{G}_{F}\right]$ with $\mathscr{X} \subseteq \mathscr{Z}$. Then (as in any lattice) $\mathscr{X} \vee(\mathscr{Y} \cap \mathscr{Z}) \subseteq(\mathscr{X} \vee \mathscr{Y}) \cap \mathscr{Z}$, so now we show the opposite inclusion. 
Take any $S \in(\mathscr{Z} \vee \mathscr{Y}) \cap \mathscr{Z}$. Then there exist $X \in \mathscr{Z}, Y \in \mathscr{Y}$, a subdirect product $T$ of $X$ and $Y$, and a morphism $\varphi$ of $T$ onto $S$.

Define $\mathscr{Z}^{\prime}=\langle X,(X \times Y) / \mu\rangle_{v}, \mathscr{Y}^{\prime}=\langle Y,(X \times Y) / \mu\rangle_{v}$ and $\mathscr{Z}^{\prime}=$ $\langle S, X,(X \times Y) / \mu\rangle_{v}$. Then $\mathscr{Z}^{\prime} \subseteq \mathscr{Z}^{\prime}$; we show that $\mathscr{Z}^{\prime}, \mathscr{Y}^{\prime}, \mathscr{Z}^{\prime}$ satisfy the modularity equation $\mathscr{X}^{\prime} \vee\left(\mathscr{Y}^{\prime} \cap \mathscr{Z}^{\prime}\right)=\left(\mathscr{X}^{\prime} \vee \mathscr{Y}^{\prime}\right) \cap \mathscr{Z}^{\prime}$.

Since $(X \times Y) / \mu \cong(X / \mu) \times(Y / \mu)$ (Result 2.2 (ii)), we see that $\mathscr{Z}^{\prime}=\langle X, Y / \mu\rangle_{v}$, and $\mathscr{Y}^{\prime}=\langle Y, X / \mu\rangle_{v}$, and by Corollary 4.4 (ii) we have that

$$
\mathscr{Z}^{\prime} \cap \mathscr{F}=\langle X / \mu, Y / \mu\rangle_{v} \cap \mathscr{F}=\mathscr{Y}^{\prime} \cap \mathscr{F} .
$$

Also $S \in\langle X \times Y\rangle_{p v}$ so $S / \mu \in\langle X \times Y\rangle_{p v}$ whence $S / \mu \in\langle X \times Y\rangle_{p v} \cap \mathscr{F}_{F}=$ $\langle(X \times Y) / \mu\rangle_{p v} \cap \mathscr{F}_{F}$ by Corollary 4.4 (i). Hence

$$
\begin{aligned}
\mathscr{Z}^{\prime} \cap \mathscr{F} & =\langle S / \mu, X / \mu,(X \times Y) / \mu\rangle_{v} \cap \mathscr{F} \\
& =\langle X / \mu,(X \times Y) / \mu\rangle_{v} \cap \mathscr{F}=\mathscr{Z}^{\prime} \cap \mathscr{F},
\end{aligned}
$$

again by Corollary 4.4(ii). Thus $\mathscr{X}^{\prime}, \mathscr{Y}^{\prime}, \mathscr{Z}^{\prime}$ all belong to the interval $\left[\left\langle\mathscr{X}^{\prime} \cap \mathscr{F}\right\rangle_{v}, \mathscr{X}^{\prime} \vee_{v} \mathscr{G}\right]$ of $\mathscr{L}_{v}(\mathscr{I})$, and this interval is modular, by Reilly's result [18, Theorem XII.2.8]. Thus

$$
S \in\left(\mathscr{Z}^{\prime} \vee_{v} \mathscr{Y}^{\prime}\right) \cap \mathscr{Z}^{\prime}=\mathscr{X}^{\prime} \vee_{v}\left(\mathscr{Y}^{\prime} \cap \mathscr{Z}^{\prime}\right)
$$

and so

$$
\begin{aligned}
S & \in \mathscr{F} \text { in } \cap\left[\mathscr{X}^{\prime} \vee_{v}\left(\mathscr{Y}^{\prime} \cap \mathscr{Z}^{\prime}\right)\right] \\
= & \left(\mathscr{F} \text { in } \cap \mathscr{X}^{\prime}\right) \vee_{p v}\left[\left(\mathscr{F} \text { in } \cap \mathscr{Y}^{\prime}\right) \cap\left(\mathscr{F} \text { in } \cap \mathscr{Z}^{\prime}\right)\right] \\
& \quad\left(\text { by Lemma } 5.1, \text { since } \mathscr{X}^{\prime}, \mathscr{Y}^{\prime}, \mathscr{Z}^{\prime} \text { are locally finite }\right) \\
= & \langle X,(X \times Y) / \mu\rangle_{p v} \vee_{p v}\left[\langle Y,(X \times Y) / \mu\rangle_{p v} \cap\langle S, X,(X \times Y) / \mu\rangle_{p v}\right]
\end{aligned}
$$

$$
\subseteq \mathscr{X} \vee_{p v}(\mathscr{Y} \cap \mathscr{Z})
$$

since $(X \times Y) / \mu \in(\mathscr{X} \vee \mathscr{Y}) \cap \mathscr{F}=\mathscr{P} \cap \mathscr{F}=\mathscr{X} \cap \mathscr{F}=\mathscr{Y} \cap \mathscr{F}=\mathscr{Z} \cap \mathscr{F}$.

Thus $\left(\mathscr{X} \vee_{p v} \mathscr{Y}\right) \cap \mathscr{Z} \subseteq \mathscr{X} \vee_{p v}(\mathscr{Y} \cap \mathscr{Z})$, which completes the proof.

We shall now show that the map $\mathscr{V} \mapsto \mathscr{V} \cap \mathscr{F}$ in of Lemma 5.1 is an isomorphism from a certain lattice of varieties to a certain lattice of pseudovarieties.

For locally finite varieties $\mathscr{V}$ and $\mathscr{W}$, since $\mathscr{V} \cap \mathscr{W}$ and $\mathscr{V} \vee_{v} \mathscr{W}$ are also locally finite, we see that the set $\mathscr{L}_{l f v}(\mathscr{U})$ of locally finite subvarieties of a variety $\mathscr{U}$ forms a (not necessarily complete) sublattice of the lattice of all subvarieties of $\mathscr{U}$.

By a locally finite pseudovariety $\mathscr{P}$ we mean one such that the generated variety $\langle\mathscr{P}\rangle_{v}$ is locally finite. It is easily seen that the set of 
locally finite pseudovarieties in a variety $\mathscr{U}$ forms a (not necessarily complete) sublattice, $\mathscr{L}_{\text {lfpv }}(\mathscr{U})$, of $\mathscr{L}_{p v}(\mathscr{U})$.

Agliano and Nation also obtained Theorem 5.3 (i) [1, Lemma 1.4(i)], and Theorem 5.3(ii) in the case where $\mathscr{U}$ is locally finite (whence $\mathscr{L}_{l f v}(\mathscr{U})=\mathscr{L}_{v}(\mathscr{U})$ and $\left.\mathscr{L}_{l f p v}(\mathscr{U})=\mathscr{L}_{p v}(\mathscr{U})\right)[1$, Lemma 1.4 (iii)].

THEOREM 5.3. (i) If $\mathscr{P}$ is a locally finite pseudovariety, then $\langle\mathscr{P}\rangle_{v} \cap$ $\mathscr{F}$ in $=\mathscr{P}$.

(ii) For any variety $\mathscr{U}$, the map $\mathscr{V} \mapsto \mathscr{V} \cap \mathscr{F}$ in is an isomorphism of $\mathscr{L}_{\text {lfv }}(\mathscr{U})$, the lattice of locally finite subvarieties of $\mathscr{U}$, to $L_{\text {lfpv }}(\mathscr{U})$, the lattice of locally finite pseudovarieties in $\mathscr{U}$. The inverse isomorphism maps each $\mathscr{P} \in L_{l f p v}(\mathscr{U})$ to $\langle\mathscr{P}\rangle_{v}$.

Proof. (i) Trivially $\mathscr{P} \subseteq\langle\mathscr{P}\rangle_{v} \cap \mathscr{F}$ in. Take any $A \in\langle\mathscr{P}\rangle_{v} \cap \mathscr{F}$ in. Then there exist algebras $C_{i}, i \in I$, in $\mathscr{P}$, a subdirect product $B$ of the $C_{i}, i \in I$, and a morphism $\varphi: B \rightarrow A$ of $B$ onto $A$. For each $a \in A$ choose an element $b_{a} \in a \varphi^{-1} \subseteq B$, and put $B^{\prime}=\left\langle\left\{b_{a} \mid\right.\right.$ $a \in A\}\rangle$, the subalgebra of $B$ generated by $\left\{b_{a} \mid a \in A\right\}$. Since $\langle\mathscr{P}\rangle_{v}$ is locally finite, $B^{\prime}$ is finite, and is a subdirect product of algebras $C_{i}^{\prime}$, $i \in I$, where each $C_{i}^{\prime}$ is a subalgebra of $C_{i}$. As $B^{\prime}$ is finite, there are essentially only finitely many morphisms from $B^{\prime}$ onto algebras (one for each congruence on $B^{\prime}$ ), so by discarding repeats, we have that $B^{\prime}$ is isomorphic to a subdirect product of finitely many $C_{i}^{\prime}$ 's. Thus $A=B^{\prime} \varphi \in \mathscr{P}$, whence $\langle\mathscr{P}\rangle_{v} \cap \mathscr{F}$ in $\subseteq \mathscr{P}$, which completes the proof.

(ii) A locally finite variety is generated by its finite members, that is, for each $\mathscr{V} \in \mathscr{L}_{l f v}(\mathscr{U})$, we have $\mathscr{V}=\langle\mathscr{V} \cap \mathscr{F} i n\rangle_{v}$. Hence the map $\mathscr{V} \mapsto$ $\mathscr{V} \cap \mathscr{F}$ in sends $\mathscr{L}_{l f v}(\mathscr{U})$ to $\mathscr{L}_{l f p v}(\mathscr{U})$, and further is one-to-one. From part (i), the map is onto. From Lemma 5.1, the map is a $\vee$-morphism, and trivially it is an $\cap$-morphism. Thus it is an isomorphism. Since $\mathscr{V}=\langle\mathscr{V} \cap \mathscr{F} i n\rangle_{v}$ for each $\mathscr{V} \in \mathscr{L}_{l f v}(\mathscr{U})$, the inverse isomorphism maps $\mathscr{P}$ to $\langle\mathscr{P}\rangle_{v}$, for each $\mathscr{P} \in \mathscr{L}_{l f p v}(\mathscr{U})$.

REMARK 5.4. For $\mathscr{P}=\mathscr{G}_{F}$, the pseudovariety of finite groups, the proof specializes to a different proof (to that of Theorem 3.3) that $\mathscr{L}_{p v}\left(\mathscr{G}_{F}\right)$ is modular, simply by deleting all mention of $(X \times Y) / \mu, X / \mu$ and $Y / \mu$ (the purpose of including $(X \times Y) / \mu$ is to put $\mathscr{Z}^{\prime}, \mathscr{Y}^{\prime}, \mathscr{Z}^{\prime}$ into a suitable subinterval of $\left.\mathscr{L}_{v}(\mathscr{I})\right)$. J. B. Nation noted that this proof applied to any modular lattice $\mathscr{L}_{v}(\mathscr{U})$ of all subvarieties of a variety $\mathscr{U}$, yielding that the lattice $\mathscr{L}_{p v}(\mathscr{U})$ of pseudovarieties in $\mathscr{U}$ is also modular. He then modified the proof (private communication) to deal 
with arbitrary term functions (not just $\mathscr{X} \vee(\mathscr{Y} \cap \mathscr{Z})$ and $(\mathscr{X} \vee \mathscr{Y}) \cap \mathscr{Z})$, thereby obtaining the result that, for any variety $\mathscr{U}$ of any algebras, $\mathscr{L}_{p v}(\mathscr{U})$ satisfies each identity satisfied by $\mathscr{L}_{v}(\mathscr{U})$ (see [1, Corollary 2.6], where this result appears with a different proof).

REMARK 5.5. C. J. Ash (private communication) generalized the just mentioned result and the result $[6,9.1]$ that the lattice of ideals $\mathscr{I}(L)$ of a lattice $L$ satisfies the same identities as $L$, by proving that an algebraic lattice satisfies the same identities as its sublattice generated by its compact elements (under finite meets and joins). To deduce [6, 9.1], merely note that the compact elements in $\mathscr{I}(L)$ are the principal ideals, and $L$ is isomorphic to its lattice of principal deals. To deduce [1, Corollary 2.6], first note that the compact elements of $\mathscr{L}_{p v}(\mathscr{U})$ are the finitely generated pseudovarieties (those generated by one finite algebra). Now from Theorem 5.3 (ii) one can show [1, Corollary 2.6]. Key results proved by Ash as part of his proof of this joint generalization were the following: (i) if $L$ is an algebraic lattice and $\left\{x_{i} \mid i \in I\right\}$ and $\left\{y_{j} \mid j \in J\right\}$ are upwardly directed subsets of $L$, then $\left(\bigvee_{i \in I} x_{i}\right) \wedge\left(\bigvee_{j \in J} x_{j}\right)=\bigvee_{i \in I, j \in J} x_{i} \wedge x_{j}$ and (ii) for any $n$-ary lattice term function $p$ and any elements $a_{1}, a_{2}, \ldots, a_{n}$ in an algebraic lattice $L, p\left(a_{1}, a_{2}, \ldots, a_{n}\right)=\bigvee\left\{p\left(c_{1}, c_{2}, \ldots, c_{n}\right) \mid\right.$ each $c_{i}$ is compact and $c_{i} \leq$ $\left.a_{i}\right\}$.

6. Meets with the pseudovariety of groups. The following lemma is analogous to the result that $\langle\mathscr{K}\rangle_{p v} \cap \mathscr{F}_{F}=\langle\mathscr{K} / \mu\rangle_{p v} \cap \mathscr{F}_{F}$ for any class $\mathscr{K}$ of finite inverse semigroups (Corollary 4.4 (i)). By Subgroups $(\mathscr{K})$ we mean the class of all groups which are subgroups of members of a class $\mathscr{K}$.

LemMa 6.1. For any class $\mathscr{K}$ of finite inverse semigroups, $\langle\mathscr{K}\rangle_{p v} \cap$ $\mathscr{G}_{F}=\langle\operatorname{Subgroups}(\mathscr{K})\rangle_{p v}$.

Proof. Of course $\langle\text { Subgroups }(\mathscr{K})\rangle_{p v} \subseteq\langle\mathscr{K}\rangle_{p v} \cap \mathscr{G}_{F}$. Take any $G \in$ $\langle\mathscr{K}\rangle_{p v} \cap \mathscr{G}_{F}$. Then there exist $C_{1}, C_{2}, \ldots, C_{n} \in \mathscr{K}$, an inverse subsemigroup $T$ of $C_{1} \times C_{2} \times \cdots \times C_{n}$, and a morphism $\varphi$ of $T$ onto $G$. Then as in [22] there exists a subgroup $H$ of $T$ such that $H \varphi=G$ (simply put $H=H_{e}$, the $\mathscr{C}$-class of $T$ containing $e$, any minimal idempotent of the subsemigroup $G \varphi^{-1}$ of $T$ ). Since $H \leq C_{1} \times C_{2} \times \cdots \times C_{n}$ we have $H \leq G_{1} \times G_{2} \times \cdots \times G_{n}$ for some groups $G_{i} \leq C_{i}, i=1,2, \ldots, n$. Thus $G \in\left\langle G_{1}, G_{2}, \ldots, G_{n}\right\rangle_{p v} \subseteq\langle\operatorname{Subgroups}(\mathscr{K})\rangle_{p v}$, whence $\langle\mathscr{K}\rangle_{p v} \cap \mathscr{G}_{F} \subseteq$ $\langle\operatorname{Subgroups}(\mathscr{K})\rangle_{p v}$, which completes the proof. 
REMARK 6.2. It is not always the case that

$$
\langle\mathscr{K}\rangle_{v} \cap \mathscr{G}=\langle\operatorname{Subgroups}(\mathscr{K})\rangle_{v},
$$

since for $\mathscr{K}$ the class of [finite] combinatorial inverse semigroups, $\langle\mathscr{K}\rangle_{v}=\mathscr{I}\left[18\right.$, Lemma XII.1.7] whence $\langle\mathscr{K}\rangle_{v} \cap \mathscr{G}=\mathscr{G}$, while $\langle\operatorname{Subgroups}(\mathscr{K})\rangle_{v}$ is the trivial variety. Likewise, not all of the analogue for varieties of the following theorem is true; see [18, Theorems XII.3.2 and Exercise XII.3.8(iv)].

TheOREM 6.3. The map $\psi$ defined by $\mathscr{P} \psi=\mathscr{P} \cap \mathscr{G}_{F}$, for each $\mathscr{P} \in$ $\mathscr{L}_{p v}\left(\mathscr{I}_{F}\right)$, is a complete lattice morphism of $\mathscr{L}_{p v}\left(\mathscr{I}_{F}\right)$ onto $\mathscr{L}_{p v}\left(\mathscr{G}_{F}\right)$. Each $\psi \circ \psi^{-1}$ congruence class is an interval of $\mathscr{L}_{p v}\left(\mathscr{I}_{F}\right)$.

Proof. Take any set $\left\{\mathscr{P}_{i} \mid i \in I\right\}$ of pseudovarieties of inverse semigroups. From Lemma 6.1, we have

$$
\begin{aligned}
\left(\bigvee_{i \in I} \mathscr{P}_{i}\right) \cap \mathscr{G}_{F} & =\left\langle\bigcup_{i \in I} \mathscr{P}_{i}\right\rangle_{p v} \cap \mathscr{G}_{F} \\
& =\left\langle\bigcup_{i \in I}\left(\mathscr{P}_{i} \cap \mathscr{G}_{F}\right)\right\rangle_{p v} \cap \mathscr{G}_{F}=\bigvee_{i \in I}\left(\mathscr{P}_{i} \cap \mathscr{G}_{F}\right)
\end{aligned}
$$

Trivially, $\left(\bigcap_{i \in I} \mathscr{P}_{i}\right) \cap \mathscr{G}_{F}=\bigcap_{i \in I}\left(\mathscr{P}_{i} \cap \mathscr{G}_{F}\right)$, so $\psi$ is a complete lattice morphism. In particular, each $\psi \circ \psi^{-1}$ class of $\mathscr{L}_{p v}\left(\mathscr{F}_{F}\right)$ is a complete sublattice of $\mathscr{L}_{p v}\left(\mathscr{I}_{F}\right)$ and hence is an interval (since $\mathscr{L}_{p v}\left(\mathscr{I}_{F}\right)$ is complete).

Clearly the interval containing each $\mathscr{P} \in \mathscr{L}_{p v}\left(\mathscr{I}_{F}\right)$ is given by

$$
\mathscr{P} \psi \circ \psi^{-1}=\left[\mathscr{P} \cap \mathscr{G}_{F}, \operatorname{Max}\left(\mathscr{P} \cap \mathscr{G}_{F}\right)\right],
$$

where $\operatorname{Max}\left(\mathscr{P} \cap \mathscr{G}_{F}\right)=\left\{S \in \mathscr{I}_{F} \mid \operatorname{Subgroups}(S) \subseteq \mathscr{P} \cap \mathscr{G}_{F}\right\}$.

REMARK 6.4. The congruence $\nu=\left(\varphi \circ \varphi^{-1}\right) \cap\left(\psi \circ \psi^{-1}\right)$ is nontrivial, which can be seen by modifying Reilly's ingenious example [18, Example XII.3.6]. Replace $\mathbf{Z}$ by $\mathbf{Z}_{n}$, the additive group of integers modulo $n$ (with $n>1$ ), and replace $\mathscr{S}$, the variety of abelian groups, by $\mathscr{A S}_{n, F}$, the pseudovariety of finite abelian groups of exponent $n$. Of course one also replaces $\mathscr{A}, \mathscr{G}$ and $\left\langle B_{2}^{1}\right\rangle$ by $\mathscr{F}_{F}, \mathscr{G}_{F}$ and $\left\langle B_{2}^{1}\right\rangle_{p v}$ respectively. These modifications also show that the lattice $\mathscr{L}_{p v}\left(\left\langle B_{2}^{1}\right\rangle_{p v} \vee \mathscr{G}_{F}\right)$ is not modular (cf. [18, Corollary XII.3.7]). 


\section{The lowest three intervals.}

Notation.

$\mathscr{T}$ denotes the class of one element (or trivial) semigroups.

$B_{2}$ denotes the five element combinatorial Brandt semigroup.

$\mathscr{B}=\left\langle B_{2}\right\rangle_{v}, \mathscr{B}_{F}=\left\langle B_{2}\right\rangle_{p v}=\mathscr{B} \cap \mathscr{F i n}$.

$\mathscr{S}=$ the variety of semilattices.

$\mathscr{S}_{F}=$ the pseudovariety of finite semilattices $=\mathscr{S} \cap \mathscr{F i n}$.

$\left[u=v, u_{i}=v_{i}\right]_{i \in \mathbf{N}}^{p v}$ denotes the pseudovariety determined by the $\omega$-sequence

$$
u=v, \quad u_{1}=v_{1}, \quad u=v, \quad u_{2}=v_{2}, \quad u=v, \ldots,
$$

in which every second identity is $u=v$.

Thus $S \in\left[u=v, u_{i}=v_{i}\right]_{i \in \mathbf{N}}^{p v}$ means that $S$ satisfies $u=v$ and, for some $n$, satisfies $u_{i}=v_{i}$ for all $i \geq n$.

Put $\mathscr{S}_{F}=\left[x x^{-1} y=y x x^{-1}\right]^{p v}$, the pseudovariety of all finite Clifford semigroups (that is, all finite semigroups which are semilattices of groups). The next theorem shows that $\mathscr{S}_{F} \vee \mathscr{G}_{F}=\mathscr{S}_{F}$; we study the modular interval $\left[\mathscr{S}_{F}, \mathscr{S}_{F}\right]$.

THEOREM 7.1. If $\mathscr{P}=\left[u_{i}=v_{i}\right]_{i \in \mathbf{N}}^{p v}$ is a group pseudovariety, then

$$
\begin{aligned}
\mathscr{P} \vee_{p v} \mathscr{S}_{F} & =\left[x x^{-1}=x^{-1} x,\left(u_{i} v_{i}^{-1}\right)^{2}=u_{i} v_{i}^{-1}\right]_{i \in \mathbf{N}}^{p v} \\
& =\text { the class of finite semilattices of groups in } \mathscr{P} \\
& =\left\{S \in \mathscr{I}_{F} \mid S \text { is a subdirect product of groups in } \mathscr{P}\right. \\
& \text { with possibly a zero adjoined }\} .
\end{aligned}
$$

Conversely, any pseudovariety $\mathscr{Q}$ consisting of Clifford semigroups and not consisting only of groups satisfies

$$
\mathscr{Q}=\left(\mathscr{Q} \cap \mathscr{G}_{F}\right) \vee_{p v} \mathscr{S}_{F} .
$$

Proof. The proofs of [18, Theorems II.2.6 and XII.4.3 and Lemma XII.4.4] also suffice for this theorem.

REMARK 7.2. Recall from $\S 1$ that $\mathscr{S}_{i c, F}$ denotes the pseudovariety of finite semigroups with idempotents commuting, and recall the following definition (for each $\mathscr{P} \in \mathscr{L}_{p v}\left(\mathscr{I}_{F}\right)$ ):

$$
\langle\mathscr{P}\rangle_{\max }=\left\{S \in \mathscr{S}_{i c, F} \mid \operatorname{Reg}(S) \subseteq \mathscr{P}\right\} .
$$

Then from Theorem 7.1, the semigroup pseudovarieties $\langle\mathscr{P}\rangle_{\max }$, for each $\mathscr{P} \in\left[\mathscr{S}_{F}, \mathscr{S}_{F}\right]$, are precisely those considered by Ash, Hall and Pin in [4], and for which the corresponding varieties of languages are found. 
COROLlaRY 7.3.

$$
\left[\mathscr{S}_{F}, \mathscr{S}_{F}\right]=\left\{\mathscr{P} \vee \mathscr{S}_{F} \mid \mathscr{P} \in \mathscr{L}_{p v}\left(\mathscr{G}_{F}\right)\right\}
$$

and

$$
\mathscr{L}_{p v}\left[\mathscr{S}_{F}\right]=\mathscr{L}_{p v}\left(\mathscr{G}_{F}\right) \cup\left[\mathscr{S}_{F}, \mathscr{S}_{F}\right] .
$$

By [18, Proposition XII.4.6] the class $\mathscr{S} \mathscr{F}$ of strict inverse semigroups (subdirect products of groups and Brandt semigroups) is a variety, determined by the identity $w w^{-1}=w^{-1} w$ where $w=y x y^{-1}$. It follows that $\mathscr{S}_{F}$, the class of finite strict inverse semigroups, is a pseudovariety, determined by the same identity; that is

$$
\mathscr{S S}_{F}=\left[w w^{-1}=w^{-1} w, w=y x y^{-1}\right]^{p v} .
$$

Likewise, from [18, Proposition XII.4.8] it follows that

$$
\begin{aligned}
\mathscr{B}_{F} & =\left[w^{2}=w, w=y x y^{-1}\right]^{p v} \\
& =\left\{S \in \mathscr{I}_{F} \mid S\right. \text { is a (finite) subdirect product of (finite) } \\
& \text { combinatorial Brandt semigroups if }|S|>1\} .
\end{aligned}
$$

For any $\omega$-sequence of identities $\left[u_{i}=v_{i}\right]_{i \in \mathbf{N}}$, there are at most $\aleph_{0}$ variables involved, say $x_{1}, x_{2}, x_{3}, \ldots$ Thus $u_{i}=v_{i}$ could be written as $u_{i}\left(x_{1}, x_{2}, x_{3}, \ldots\right)=v_{i}\left(x_{1}, x_{2}, x_{3}, \ldots\right)$, though of course only finitely many of $x_{1}, x_{2}, x_{3}, \ldots$ actually occur in the two words $u_{i}$ and $v_{i}$. We write $\left(x_{k}\right)=\left(x_{1}, x_{2}, x_{3}, \ldots\right)$ and $u_{i}\left(x_{k}\right)=v_{i}\left(x_{k}\right)$ for $u_{i}\left(x_{1}, x_{2}, x_{3}, \ldots\right)=$ $v_{i}\left(x_{1}, x_{2}, x_{3}, \ldots\right)$.

As in [18], for any inverse semigroup word $w$, the notation $w \in G$ means the identity $w w^{-1}=w^{-1} w$, and $w \in E$ means the identity $w^{2}=w$.

THEOREM 7.4. If $\mathscr{P}=\left[u_{i}\left(x_{k}\right)=v_{i}\left(x_{k}\right)\right]_{i \in \mathbf{N}}$ is any group pseudovariety, then

$$
\begin{aligned}
\mathscr{P} \vee_{p v} \mathscr{B}_{F}= & {\left[y x y^{-1} \in G, u_{i}\left(y_{k} x_{k} y_{k}^{-1}\right)\left[v_{i}\left(y_{k} x_{k} y_{k}^{-1}\right)\right]^{-1} \in E\right]_{i \in \mathbf{N}} } \\
= & \left\{S \in \mathscr{S} \mathscr{I}_{F} \mid \operatorname{Subgroups}(S) \subseteq \mathscr{P}\right\} \\
= & \left\{S \in \mathscr{I}_{F} \mid S \text { is a (finite) subdirect product of groups in } \mathscr{P}\right. \\
& \quad \text { and (finite) Brandt semigroups over groups in } \mathscr{P}\} .
\end{aligned}
$$

Proof. The proof of [18, Theorem XII.4.10] suffices also to prove Theorem 7.4. Note that in particular $\mathscr{G}_{F} \vee_{p v} \mathscr{B}_{F}=\mathscr{S}_{F}$.

The following analogue of [18, Proposition XII.4.13] is easily proved ( $Y_{2}$ denotes the two element semilattice, and a strict pseudovariety means one containing only strict inverse semigroups). 
THEOREM 7.5. For any $\mathscr{P} \in \mathscr{L}_{p v}\left(\mathscr{I}_{F}\right)$,

(i) $\mathscr{P}$ is a group pseudovariety if and only if $Y_{2} \notin \mathscr{P}$,

(ii) $\mathscr{P}$ is a Clifford pseudovariety if and only if $B_{2} \notin \mathscr{P}$,

(iii) $\mathscr{P}$ is a strict pseudovariety if and only if $B_{2}^{1} \notin \mathscr{P}$.

COROLlaRY 7.6. In the lattice $\mathscr{L}_{p v}\left(\mathscr{J}_{F}\right)$,

(i) $\mathscr{S}_{F}$ is the least nongroup pseudovariety,

(ii) $\mathscr{B}_{F}$ is the least non-Clifford pseudovariety,

(iii) $\left\langle B_{2}^{1}\right\rangle_{p v}$ is the least nonstrict pseudovariety,

(iv) $\mathscr{T} \prec \mathscr{S}_{F} \prec \mathscr{B}_{F} \prec\left\langle B_{2}^{1}\right\rangle_{p v}$.

Note that from Corollary 7.6 (ii) and (iv), we have that $\mathscr{L}_{p v}\left(\mathscr{B}_{F}\right)$ is the three element chain.

LemMa 7.7. Let $\mathscr{P}$ be a strict pseudovariety which is not a Clifford pseudovariety. Then $\mathscr{P}=\left(\mathscr{P} \cap \mathscr{G}_{F}\right) \vee \mathscr{B}_{F}$.

Proof. The proof of [18, Lemma XII.4.15] also suffices to prove Lemma 7.7.

THEOREM 7.8. The function $\chi: \mathscr{L}_{p v}\left(\mathscr{S}_{F}\right) \rightarrow \mathscr{L}_{p v}\left(\mathscr{B}_{F}\right) \times \mathscr{L}_{p v}\left(\mathscr{G}_{F}\right)$, given by $\mathscr{P} \chi=\left(\mathscr{P} \cap \mathscr{B}_{F}, \mathscr{P} \cap \mathscr{G}_{F}\right)$ for each $\mathscr{P} \in \mathscr{L}_{p v}\left(\mathscr{S}_{F}\right)$, is a lattice isomorphism.

Proof. The proof of [18, Theorem XII.4.16] also suffices to prove Theorem 7.8.

CoROllary 7.9. The lattice $\mathscr{L}_{p v}\left(\mathscr{S}_{F}\right)$ is modular.

Proof. Now $\mathscr{L}_{p v}\left(\mathscr{G}_{F}\right)$ is modular, by Theorem 3.3 or Theorem 5.2, and the three element chain $\mathscr{L}_{p v}\left(\mathscr{B}_{F}\right)$ is modular, so $\mathscr{L}_{p v}\left(\mathscr{B}_{F}\right) \times \mathscr{L}_{p v}\left(\mathscr{G}_{F}\right)$ $\cong \mathscr{L}_{p v}\left(\mathscr{S S}_{F}\right)$ is also modular.

\section{REFERENCES}

[1] P. Agliano and J. B. Nation, Lattices of pseudovarieties, J. Austral. Math. Soc. (Series A), to appear.

[2] C. J. Ash, Pseudovarieties, generalized varieties, and similarly described classes, J. Algebra, 92 (1985), 104-115.

[3] C. J. Ash, Finite semigroups with commuting idempotents, J. Austral. Math. Soc. (Series A), 43 (1987), 81-90. 
[4] C. J. Ash, T. E. Hall and J. E. Pin, On the varieties of languages associated with some varieties of finite monoids with commuting idempotents, submitted.

[5] J. C. Birget, S. W. Margolis and J. Rhodes, Finite semigroups whose idempotents commute or form a subsemigroup, Proceedings of the 1986 Chico conference on semigroups, D. Reidel, (1987), 25-36.

[6] P. Crawley and R. P. Dilworth, Algebraic Theory of Lattices, Prentice Hall, Englewood Cliffs, N. J., 1973.

[7] S. Eilenberg, Automata, Languages and Machines, Vols. A, B, Academic Press, London, New York, 1974, 1976.

[8] S. Eilenberg and M. P. Schützenberger, On pseudovarieties, Adv. in Math., 19 (1976), 413-418.

[9] T. E. Hall, On regular semigroups, J. Algebra, 24 (1973), 1-24.

[10] _ Biprefix codes, inverse semigroups and syntactic monoids of injective automata, Theoret. Comp. Sci., 32 (1984), 201-213.

[11] J. M. Howie, An Introduction to Semigroup Theory, London Math. Society Monographs 7 (Academic Press, London, New York, 1976).

[12] M. Keenan and G. Lallement, On certain codes admitting inverse semigroups as syntactic monoids, Semigroup Forum, 8 (1974), 312-331.

[13] G. Lallement, Semigroups and Combinatorial Applications, Wiley, New York, 1979.

[14] S. W. Margolis and J. E. Pin, Languages and inverse semigroups, 11 th ICALP, pp. 337-346 Lecture Notes in Computer Science 172, 1984.

[15] _ Graphs, inverse semigroups and languages, Proceedings of 1984 Marquette conference on semigroups, pp. 85-112.

[16] - Finite inverse semigroups, varieties and languages, J. Algebra, to appear.

[17] Hanna Neumann, Varieties of Groups, Ergeb. Math. Grenzgeb., Springer, Berlin (1967).

[18] Mario Petrich, Inverse Semigroups, (John Wiley \& Sons, 1984).

[19] J. E. Pin, On the languages accepted by finite reversible automata, submitted.

[20] N. R. Reilly, Modular sublattices of the lattice of varieties of inverse semigroups, Pacific J. Math., 89 (1980), 405-417.

[21] Ch. Reutenauer, Une topologie du monoide libre, Semigroup Forum, 18 (1979), 33-49, and Sur mon article une topologie du monoide libre, Semigroup Forum, 22 (1981), 93-95.

[22] J. R. Rhodes, Some results on finite semigroups, J. Algebra, 3 (1966), 471-504.

Received November 12, 1987. This work was supported by research grants from the College of Charleston and the Australian Research Grants Commission.

Monash University

Clayton, Victoria 3168

Australia

AND

College of Charleston

Charleston, SC 29424 